\title{
Effect of the Financial Crisis on Socioeconomic Inequalities in Mortality in Small Areas in Seven Spanish Cities
}

\author{
Mercè Gotsens ${ }^{1,2} \mathbb{E}$, Josep Ferrando ${ }^{1}$, Marc Marí-Dell'Olmo ${ }^{1,2,3} \mathbb{C}$, Laia Palència ${ }^{1,2,3}$, \\ Xavier Bartoll ${ }^{1,2}$, Ana Gandarillas ${ }^{4}$, Pablo Sanchez-Villegas ${ }^{3,5}$, Santi Esnaola ${ }^{6}$, \\ Antonio Daponte ${ }^{3,5}$ and Carme Borrell 1,2,3,7,* \\ 1 Agència de Salut Pública de Barcelona, 08023 Barcelona, Spain \\ 2 Institut d'Investigació Biomèdica (IIB Sant Pau), 08041 Barcelona, Spain \\ 3 CIBER Epidemiología y Salud Pública (CIBERESP), 28029 Madrid, Spain \\ 4 Dirección General de Salud Pública, Consejería de Sanidad, Comunidad de Madrid, 28035 Madrid, Spain \\ 5 Observatorio de Salud y Medio Ambiente de Andalucía, Escuela Andaluza de Salud Pública, \\ 18080 Granada, Spain \\ 6 Department of Health of the Basque Country, 01006 Vitoria-Gasteiz, Spain \\ 7 Department of Experimental and Health Sciences, Universitat Pompeu Fabra, 08002 Barcelona, Spain \\ * Correspondence: cborrell@aspb.cat
}

Received: 9 December 2019; Accepted: 30 January 2020; Published: 4 February 2020

\begin{abstract}
Background: The aim of this study was to analyze the trend in socioeconomic inequalities in mortality in small areas due to several specific causes before (2001-2004, 2005-2008) and during (2009-2012) the economic crisis in seven Spanish cities. Methods: This ecological study of trends, with census tracts as the areas of analysis, was based on three periods. Several causes of death were studied. A socioeconomic deprivation index was calculated for each census tract. For each small area, we estimated standardized mortality ratios, and controlled for their variability using Bayesian models (sSMR). We also estimated the relative risk of mortality according to deprivation in the different cities, periods, and sexes. Results: In general, a similar geographical pattern was found for the socioeconomic deprivation index and sSMR. For men, there was an association in all cities between the deprivation index and all-cause mortality that remained stable over the three periods. For women, there was an association in Barcelona, Granada, and Sevilla between the deprivation index and all-cause mortality in the third period. Patterns by causes of death were more heterogeneous. Conclusions: After the start of the financial crisis, socioeconomic inequalities in total mortality in small areas of Spanish cities remained stable in most cities, although several causes of death showed a different pattern.
\end{abstract}

Keywords: socioeconomic factors; trends; mortality; inequalities; urban areas; small areas

\section{Introduction}

The impact of economic crises on populations' health depends on various factors, such as the institutional, cultural, and socioeconomic context of the society, the severity and duration of the crisis, and the responses articulated to combat it [1-7]. Crises may be accompanied by a higher prevalence of poor health or premature mortality, particularly in vulnerable population subgroups [8,9]. However, some research has described how recessions might improve health, at least in the short term, with a pro-cyclical worsening of mortality during periods of growth and improvement during recessions, mainly due to fewer traffic injuries and alcohol-related deaths [10]. Catalano et al. (2011) [11] described the mechanisms related to changes in health outcomes during economic recessions, most of which are 
due to job loss and financial strain, as well as stress, frustration, and aggression, and the investment of time and effort in managing the sequelae of job or income loss.

In the European Union, the financial crisis started in 2008. During this period, no change was observed in either all-cause mortality or its decreasing tendency [12-14]. However, mortality due to suicide increased [12-17], while mortality due to traffic injuries decreased [12-14,17]. In Spain, mortality continued to decrease at the start of the crisis for most causes [18].

Financial crises usually affect the most deprived populations because they have a higher probability of job and even housing loss, given the high number of evictions in Spain during the crisis [19]. Studies analyzing the effects of the financial crisis on inequalities in health have reported heterogeneous results. Inequalities have increased in some countries, but have remained stable in others $[4,12,20]$. In Spain, the crisis has had the strongest impact in the most disadvantaged population groups and inequalities in health have increased, depending on the health indicator analyzed [7,21,22].

Few studies have analyzed the impact of the financial crisis on health at the city level, where socioeconomic inequalities in mortality in small areas of cities have been described, with the most disadvantaged areas showing the highest mortality [23-28]. Local governments in Spain have no responsibility in the management of healthcare services; however, they are responsible for fundamental aspects of social and health protection that can improve the health of the population, and especially, reduce health inequalities.

Thus, the aim of this study was to analyze the trend in socio-economic inequalities in mortality in small areas due to several specific causes before (2001-2004, 2005-2008) and during (2009-2012) the economic crisis in seven Spanish cities.

\section{Methods}

\subsection{Design, Unit of Analysis, and Study Population}

This ecological study of trends was based on three periods (2001-2004, 2005-2008, and 2009-2012) and forms part of the IMCRISES project (the effect of the economic crisis on mortality, reproductive health, and health inequalities in Spain) [29]. The units of analysis were the census tracts of seven Spanish cities, as defined in the 2001 Spanish Population and Housing Census. These cities vary in size and are located in different geographical regions of Spain (Center, South, North, and East). The study population consisted of the residents of the seven cities during the period 2001-2012.

\subsection{Information Sources}

Mortality data grouped by sex, census tract, and period were obtained from the mortality registers of the corresponding cities or autonomous communities (Spanish regions). The census tract was obtained from the postal address of the deceased provided on the death certificate or from the Register of Inhabitants of each city. Population data stratified by age (in 5-year groups), sex, census tract, and period were obtained from the Register of Inhabitants for each city or from the National Institute of Statistics (Instituto Nacional de Estadística). The 2001 Population and Housing Census was used to obtain the information needed to construct the socioeconomic deprivation index for each city [30].

\subsection{Mortality}

This study analyzed all-cause mortality and deaths due to several specific causes, namely, infectious and parasitic diseases, diabetes mellitus, ischemic heart disease, cerebrovascular diseases, cirrhosis, suicide, and traffic injury. These causes of death are those most strongly affected by economic crises $[4,8,12,20]$. The underlying cause of death was coded using the International Classification of Diseases, tenth revision (ICD-10) (see the codes in the footnote to Table 1). 


\subsection{Socioeconomic Deprivation Index}

We included a socioeconomic deprivation index as a covariate, which was the first component of a principal components analysis performed within each city for five socioeconomic indicators corresponding to 2001 and available for each census tract, based on the methodology described by Domínguez-Berjón et al. [31]. The indicators included in the index were the percentages of: (a) Unemployment: percentage of people $\geq 16$ years or over who were unemployed or actively seeking a job in relation to the total economically active population; (b) low educational level (primary school or lower): percentage of people $\geq 16$ years with less than 5 years of schooling or with 5 years of schooling or more who did not complete basic compulsory education, in relation to the total population $\geq 16$ years; (c) low educational level in young people (16-29 years); (d) manual workers: percentage of people $\geq 16$ years, in manual employment in relation to the total employed population $\geq 16$ years; and (e) temporary workers: Percentage of people $\geq 16$ years, employed in temporary jobs, in relation to the total employed population $\geq 16$ years. A socioeconomic deprivation index was obtained for each city. Each index was normalized to achieve a mean of 0 and standard deviation of 1 . In all cities, the index accounted for over $75 \%$ of the variability of the included indicators.

\subsection{Data Analysis}

Age-standardized mortality rates (ASMR) were calculated by the direct method using the Spanish population for the year 2001 as the reference population. ASMR were calculated for each cause of death, sex, period, and city.

For small areas, the mortality indicator used for the analysis was the standardized mortality ratio (SMR). The SMR is dependent on population size since its variance is inversely proportional to the expected values; thus, areas with a small population tend to show highly variable estimates. To smooth the SMR, we used the hierarchical Bayesian model proposed by Besag, York, and Mollié [32]. This model takes two types of random effects into account, spatial and heterogeneous: the former takes account of the spatial structure of the data, while the latter deals with non-structural (non-spatial) variability. Smoothed SMR (sSMR) were estimated for each cause of death, period, and city with the following model:

- $O_{i} \sim \operatorname{Poisson}\left(E_{i} \theta_{i}\right)$

- $\log \left(\theta_{i}\right)=\alpha+S_{i}+H_{i}($ model 1$)$

where, for each area $i, O_{i}$ is the number of observed cases, $E_{i}$ the expected cases, $\theta_{i}$ the sSMR with respect to the whole city, $S_{i}$ the spatial effect, and $H_{i}$ the heterogeneous effect. The expected cases were calculated by indirect standardization taking as reference the mortality rates of each city by age (in five-year groups), cause of death, and period.

The geographical distribution of the SSMR is represented using maps of septiles. The deprivation index results are also represented as septile maps. All maps were generated using the R statistical package [33].

To analyze the relationship between socioeconomic deprivation and mortality in the three periods, we fitted an ecological regression model for each city that included the deprivation index (D), the period (though two dummy variables $P_{2}$ and $P_{3}$ ), and their interaction:

- $O_{i t} \sim \operatorname{Poisson}\left(E_{i t} \theta_{i t}\right)$

- $\log \left(\theta_{i t}\right)=\alpha+\beta_{1} D_{1}+\beta_{2} P_{2 t}+\beta_{3} P_{3 t}+\beta_{4} P_{2 t} D_{i}+\beta_{5} P_{3 t} D_{t}+S_{i t}+H_{i t}$ (model 2)

where, for each area $i$ and period $t(t=1$ for period 2001-2004, $t=2$ for period 2005-2008 and $t=3$ for period 2009-2012), $O_{i t}$ is the number of observed cases, $E_{i t}$ the expected cases, $\theta_{i t}$ the sSMR with respect to the whole city, $S_{i t}$ the spatial effect, and $H_{i t}$ the heterogeneous effect. Finally, two dummy variables $P_{2 t}$ and $P_{3 t}$ take the following values: $P_{j t}=1$ if $j=\mathrm{t}$ and $P_{j t}=0$ otherwise. The expected cases were calculated taking as reference the mortality rates of the first period (2001-2004). Thus, the risk of mortality associated with the deprivation index was calculated as $\exp \left(\beta_{1}\right)$ in the first period, as $\exp \left(\beta_{1}\right.$ 
$\left.+\beta_{4}\right)$ in the second period, and as $\exp \left(\beta_{1}+\beta_{5}\right)$ in the third period. Changes between periods in the relationship between the socioeconomic deprivation index and mortality were evaluated through the interactions included in model 2. Specifically, we studied the change between the first and second period ( $\beta 4)$ and the second and third periods ( $\beta 5-\beta 4)$.

In the two models, an intrinsic conditional autoregressive prior distribution (ICAR) [32] was assigned to the spatial effect, which assumed that the expected value of each area coincided with the mean of the spatial effect of the adjacent areas and had a variance of $\sigma_{\mathrm{Sr}^{\prime}}^{2}$, while the heterogeneous effect was represented by using a normal distribution with mean 0 and variance $\sigma_{\mathrm{Hr}}^{2}$. A half-normal distribution with mean 0 and precision 0.001 was assigned to the standard deviation $\sigma_{\mathrm{Sr}}$ and $\sigma_{\mathrm{H}}$. A normal vague prior distribution was assigned to the parameters $\alpha, \beta_{1}, \ldots, \beta_{5}$.

As the deprivation index scale is dimensionless and arbitrarily fixed, for each cause of death and city, we calculated the increase in risk corresponding to a change in deprivation index from its 95th percentile value $\left(\mathrm{P}_{95}\right)$ (severe deprivation) to its 5 th percentile value $\left(\mathrm{P}_{5}\right)$ (low deprivation).

Relative risk (RR) estimates were obtained based on the mean of their posterior distribution, along with the corresponding 95\% credible intervals $(95 \% \mathrm{CI})$. All distributions were obtained using the "Integrated nested Laplace approximations" method (INLA), using the INLA library of the R statistical package, R.3.5.0. [34].

Finally, estimation of RRs for suicide and traffic injuries was not feasible for San Sebastian and Granada due to the small number of deaths among women.

\section{Results}

Tables 1 and 2 show, for each city and period the population, the number of deaths and the age-standardized mortality rate (ASMR) by cause of death for men and women, respectively. In general, the all-cause and cause-specific mortality rates decreased in the second and third periods in most of the cities in both men and women. For example, in Madrid, in the first period, the ASMR for all causes was 1112.1 per 100,000 inhabitants, which fell to 883.9 in the third period among men. In women, the rates were 581.0 in the first period and 492.0 in the third.

In general, the geographical pattern was similar for the socioeconomic deprivation index and sSMR. For example, the Figure 1 shows the geographical distribution of the socioeconomic deprivation index and the distribution, in the three periods, of sSMR for cirrhosis among men in Barcelona and of sSMR for ischemic heart disease among women in Sevilla. Green areas represent low sSMR and low socioeconomic deprivation, while brown areas represent high sSMR and high socioeconomic deprivation. 
Table 1. Population, number of deaths (N), and age-standardized mortality rate (ASMR) per 100,000 inhabitants by period and cause of death in 7 Spanish cities for men, 2001-2012.

\begin{tabular}{|c|c|c|c|c|c|c|c|c|c|c|c|c|c|c|c|c|c|c|}
\hline \multirow[b]{2}{*}{$\begin{array}{c}\text { Cities (No. Census } \\
\text { Tract) }\end{array}$} & \multirow[b]{2}{*}{ Period } & \multirow[b]{2}{*}{ Pob } & \multicolumn{2}{|c|}{$\begin{array}{c}\text { Infectious and } \\
\text { Parasitic Diseases }\end{array}$} & \multicolumn{2}{|c|}{ Diabetes } & \multicolumn{2}{|c|}{$\begin{array}{l}\text { Ischemic Heart } \\
\text { Diseases }\end{array}$} & \multicolumn{2}{|c|}{$\begin{array}{l}\text { Cerebrovascular } \\
\text { Diseases }\end{array}$} & \multicolumn{2}{|c|}{ Cirrhosis } & \multicolumn{2}{|c|}{ Suicide } & \multicolumn{2}{|c|}{ Traffic Injuries } & \multicolumn{2}{|c|}{ All Causes } \\
\hline & & & $\mathbf{N}$ & ASMR & $\mathbf{N}$ & ASMR & $\mathbf{N}$ & ASMR & $\mathbf{N}$ & ASMR & $\mathbf{N}$ & ASMR & $\mathbf{N}$ & ASMR & $\mathbf{N}$ & ASMR & $\mathbf{N}$ & ASMR \\
\hline \multirow[t]{3}{*}{ Barcelona (1491) } & $2001-2004$ & 293,6440 & 799 & 26.9 & 725 & 25.0 & 3565 & 122.3 & 2187 & 77.4 & 672 & 21.8 & 342 & 11.4 & 285 & 9.7 & 32,128 & 1105.9 \\
\hline & 2005-2008 & 304,6533 & 768 & 24.3 & 702 & 22.6 & 3047 & 98.5 & 1957 & 63.8 & 553 & 17.4 & 339 & 10.6 & 195 & 6.5 & 31,247 & 1011.1 \\
\hline & $2009-2012$ & 308,3469 & 609 & 18.1 & 648 & 18.9 & 2835 & 84.4 & 1658 & 48.6 & 557 & 17.1 & 332 & 10.2 & 134 & 4.3 & 29,724 & 883.9 \\
\hline \multirow[t]{3}{*}{ Bilbao (288) } & 2001-2004 & 657,840 & 174 & 26.5 & 129 & 21.7 & 698 & 113.0 & 549 & 91.4 & 174 & 25.0 & 78 & 11.5 & 84 & 13.0 & 7282 & 1177.0 \\
\hline & 2005-2008 & 658,272 & 177 & 25.0 & 131 & 19.0 & 710 & 101.5 & 530 & 77.9 & 161 & 22.2 & 82 & 11.5 & 46 & 7.0 & 7350 & 1065.5 \\
\hline & 2009-2012 & 660,882 & 119 & 15.1 & 145 & 18.7 & 729 & 94.0 & 470 & 61.3 & 152 & 19.5 & 69 & 9.8 & 43 & 6.3 & 7392 & 963.3 \\
\hline \multirow[t]{3}{*}{ San Sebastian (140) } & 2001-2004 & 333,822 & 81 & 26.1 & 66 & 23.7 & 318 & 106.5 & 226 & 79.3 & 76 & 22.8 & 37 & 11.3 & 53 & 16.1 & 3346 & 1131.8 \\
\hline & 2005-2008 & 336,108 & 68 & 19.9 & 63 & 18.9 & 283 & 84.4 & 217 & 66.5 & 88 & 23.6 & 42 & 11.8 & 38 & 11.0 & 3419 & 1018.2 \\
\hline & 2009-2012 & 339,319 & 51 & 13.6 & 82 & 22.6 & 271 & 72.1 & 221 & 60.0 & 59 & 15.0 & 34 & 9.6 & 17 & 5.1 & 3353 & 904.6 \\
\hline \multirow[t]{3}{*}{ Vitoria (168) } & 2001-2004 & 429,826 & 89 & 25.5 & 58 & 19.4 & 282 & 88.3 & 233 & 80.4 & 84 & 21.7 & 59 & 14.4 & 86 & 20.9 & 3426 & 1074.5 \\
\hline & 2005-2008 & 451,026 & 82 & 21.4 & 84 & 23.3 & 288 & 75.0 & 227 & 63.1 & 100 & 23.5 & 66 & 15.1 & 49 & 11.4 & 3680 & 971.6 \\
\hline & 2009-2012 & 468,668 & 59 & 12.9 & 75 & 17.6 & 306 & 68.9 & 206 & 48.1 & 94 & 20.0 & 62 & 12.6 & 24 & 5.1 & 3808 & 864.0 \\
\hline \multirow[t]{3}{*}{ Madrid (2358) } & 2001-2004 & $5,498,348$ & 1235 & 23.4 & 664 & 14.3 & 5788 & 119.4 & 3037 & 64.9 & 1056 & 19.8 & 432 & 7.9 & 554 & 9.9 & 54,053 & 1112.1 \\
\hline & 2005-2008 & $5,668,478$ & 1132 & 20.1 & 595 & 11.6 & 5262 & 100.2 & 2810 & 54.4 & 915 & 16.4 & 240 & 4.1 & 355 & 6.2 & 53,087 & 1008.3 \\
\hline & 2009-2012 & $5,776,884$ & 1098 & 18.3 & 649 & 11.2 & 4427 & 76.6 & 2349 & 40.7 & 728 & 12.4 & 126 & 2.1 & 107 & 1.8 & 50,979 & 883.9 \\
\hline \multirow[t]{3}{*}{ Granada (181) } & 2001-2004 & 441,668 & 114 & 28.4 & 78 & 21.3 & 599 & 164.2 & 348 & 99.2 & 130 & 31.9 & 56 & 13.2 & 69 & 15.0 & 4285 & 1169.1 \\
\hline & $2005-2008$ & 439,300 & 118 & 28.3 & 90 & 23.5 & 509 & 132.0 & 345 & 91.2 & 143 & 34.1 & 50 & 11.7 & 53 & 12.0 & 4409 & 1128.9 \\
\hline & 2009-2012 & 446,516 & 72 & 15.8 & 103 & 23.7 & 536 & 122.1 & 298 & 69.2 & 101 & 22.6 & 88 & 19.6 & 22 & 4.9 & 4217 & 969.3 \\
\hline \multirow[t]{3}{*}{ Sevilla (510) } & 2001-2004 & $1,357,036$ & 292 & 24.3 & 169 & 17.9 & 1808 & 188.6 & 1398 & 161.7 & 321 & 27.3 & 145 & 11.5 & 154 & 11.1 & 11,655 & 1193.8 \\
\hline & $2005-2008$ & $1,332,728$ & 318 & 26.4 & 182 & 18.1 & 1463 & 141.9 & 1309 & 137.0 & 319 & 26.1 & 130 & 10.2 & 104 & 7.8 & 12,017 & 1155.8 \\
\hline & 2009-2012 & $1,337,716$ & 260 & 20.8 & 193 & 18.1 & 1242 & 110.7 & 1002 & 94.6 & 323 & 25.1 & 115 & 9.0 & 56 & 4.3 & 11,836 & 1051.5 \\
\hline
\end{tabular}

International Classification of Diseases (ICD-10): Infectious and parasitic diseases (A00-B99, R75); Diabetes (E10-E14); Ischemic heart diseases (I20-I25), Cerebrovascular diseases (I60-I69), Cirrhosis (K70, K72.1, K73, K74, K76(.1.9)); Suicide (X60-X84); Traffic injuries (V02-V04(.1.9), V09(.2.3), V12-V14(.3.4.5.9), V19(.4.5.6.9), V20-V28(.3.4.5.9), V29-V79(.4.5.6.7.8.9), V80(.3.4.5),

V81-V82(.1), V83-V86(.0.1.2.3), V87(.0.1.2.3.4.5.6.7.8), V89(.2.9)). 
Table 2. Population, number of deaths (N), and age-standardized mortality rate (ASMR) per 100,000 inhabitants by period and cause of death in 7 Spanish cities for women, 2001-2012.

\begin{tabular}{|c|c|c|c|c|c|c|c|c|c|c|c|c|c|c|c|c|c|c|}
\hline \multirow[b]{2}{*}{$\begin{array}{l}\text { Cities (No. Census } \\
\text { Tract) }\end{array}$} & \multirow[b]{2}{*}{ Period } & \multirow[b]{2}{*}{ Pob } & \multicolumn{2}{|c|}{$\begin{array}{c}\text { Infectious and } \\
\text { Parasitic Diseases }\end{array}$} & \multicolumn{2}{|c|}{ Diabetes } & \multicolumn{2}{|c|}{$\begin{array}{c}\text { Ischemic Heart } \\
\text { Diseases }\end{array}$} & \multicolumn{2}{|c|}{$\begin{array}{l}\text { Cerebrovascular } \\
\text { Diseases }\end{array}$} & \multicolumn{2}{|c|}{ Cirrhosis } & \multicolumn{2}{|c|}{ Suicide } & \multicolumn{2}{|c|}{ Traffic Injuries } & \multicolumn{2}{|c|}{ All Causes } \\
\hline & & & $\mathbf{N}$ & ASMR & $\mathbf{N}$ & ASMR & $\mathbf{N}$ & ASMR & $\mathbf{N}$ & ASMR & $\mathbf{N}$ & ASMR & $\mathbf{N}$ & ASMR & $\mathbf{N}$ & ASMR & $\mathbf{N}$ & ASMR \\
\hline \multirow[t]{3}{*}{ Barcelona (1491) } & $2001-2004$ & $3,298,056$ & 723 & 15.2 & 959 & 17.0 & 2878 & 49.8 & 3441 & 59.0 & 443 & 9.6 & 163 & 4.4 & 146 & 4.0 & 33,501 & 614.0 \\
\hline & $2005-2008$ & $3,375,674$ & 749 & 14.0 & 885 & 14.2 & 2336 & 37.2 & 2949 & 46.1 & 409 & 8.7 & 157 & 4.1 & 67 & 1.8 & 32,914 & 557.0 \\
\hline & 2009-2012 & $3,406,659$ & 655 & 10.9 & 789 & 11.5 & 2102 & 29.8 & 2510 & 35.7 & 381 & 7.8 & 159 & 4.3 & 62 & 1.5 & 32,306 & 501.6 \\
\hline \multirow[t]{3}{*}{ Bilbao (288) } & 2001-2004 & 730,202 & 136 & 14.3 & 177 & 15.6 & 504 & 44.6 & 731 & 64.2 & 94 & 9.9 & 29 & 3.6 & 42 & 5.5 & 6935 & 636.7 \\
\hline & $2005-2008$ & 734,570 & 134 & 11.9 & 189 & 14.4 & 463 & 35.3 & 691 & 52.2 & 61 & 6.0 & 50 & 6.0 & 17 & 1.7 & 7050 & 567.0 \\
\hline & 2009-2012 & 738,067 & 147 & 10.8 & 167 & 10.6 & 447 & 29.6 & 645 & 41.6 & 76 & 7.0 & 33 & 3.7 & 9 & 1.2 & 7224 & 502.4 \\
\hline \multirow[t]{3}{*}{ San Sebastian (140) } & 2001-2004 & 375,249 & 68 & 11.7 & 89 & 14.7 & 254 & 40.9 & 399 & 65.4 & 30 & 6.0 & 29 & 6.7 & 23 & 5.1 & 3664 & 619.8 \\
\hline & $2005-2008$ & 380,058 & 70 & 10.7 & 72 & 10.7 & 224 & 31.8 & 361 & 51.8 & 26 & 4.9 & 8 & 2.1 & 13 & 3.0 & 3625 & 538.3 \\
\hline & 2009-2012 & 385,302 & 50 & 6.5 & 93 & 11.8 & 191 & 22.4 & 308 & 37.5 & 35 & 6.1 & 18 & 3.4 & 9 & 1.7 & 3824 & 504.8 \\
\hline \multirow[t]{3}{*}{ Vitoria (168) } & $2001-2004$ & 443,772 & 71 & 14.6 & 87 & 17.6 & 203 & 40.7 & 294 & 58.0 & 36 & 7.9 & 16 & 3.6 & 23 & 5.1 & 3094 & 622.6 \\
\hline & $2005-2008$ & 463,781 & 66 & 11.8 & 117 & 18.7 & 209 & 34.6 & 269 & 43.3 & 29 & 5.7 & 16 & 3.2 & 13 & 2.5 & 3231 & 541.2 \\
\hline & 2009-2012 & 482,422 & 50 & 6.9 & 100 & 12.9 & 227 & 28.8 & 261 & 35.2 & 35 & 6.0 & 12 & 2.2 & 11 & 2.5 & 3417 & 475.9 \\
\hline \multirow[t]{3}{*}{ Madrid (2358) } & 2001-2004 & $6,273,028$ & 932 & 11.3 & 1038 & 10.9 & 4949 & 51.9 & 4762 & 49.8 & 589 & 7.4 & 193 & 2.8 & 230 & 3.6 & 52,832 & 581.0 \\
\hline & $2005-2008$ & $6,437,031$ & 1017 & 11.1 & 901 & 8.6 & 4532 & 43.2 & 4266 & 40.7 & 405 & 4.9 & 95 & 1.4 & 141 & 2.1 & 54,214 & 544.5 \\
\hline & 2009-2012 & $6,599,904$ & 1045 & 10.0 & 983 & 8.1 & 3934 & 33.0 & 3758 & 31.5 & 361 & 4.0 & 34 & 0.5 & 41 & 0.6 & 54,534 & 492.0 \\
\hline \multirow[t]{3}{*}{ Granada (181) } & 2001-2004 & 508,984 & 63 & 10.7 & 102 & 16.3 & 473 & 74.8 & 481 & 74.6 & 76 & 13.1 & 27 & 5.1 & 19 & 3.6 & 4206 & 678.1 \\
\hline & $2005-2008$ & 505,528 & 93 & 14.2 & 103 & 14.7 & 435 & 60.7 & 499 & 68.9 & 54 & 8.6 & 36 & 6.6 & 10 & 2.0 & 4547 & 658.1 \\
\hline & 2009-2012 & 513,880 & 60 & 8.0 & 113 & 14.3 & 574 & 68.3 & 430 & 51.8 & 52 & 8.1 & 27 & 4.9 & 6 & 1.3 & 4561 & 581.9 \\
\hline \multirow{3}{*}{ Sevilla (510) } & 2001-2004 & $1,482,864$ & 181 & 11.3 & 270 & 15.9 & 1457 & 85.6 & 2583 & 147.7 & 112 & 7.2 & 48 & 3.1 & 45 & 3.1 & 11,545 & 684.0 \\
\hline & $2005-2008$ & $1,463,852$ & 188 & 11.0 & 310 & 16.8 & 1101 & 59.9 & 2207 & 115.7 & 115 & 7.1 & 51 & 3.3 & 23 & 1.6 & 11,805 & 650.7 \\
\hline & 2009-2012 & $1,474,368$ & 194 & 10.3 & 232 & 10.8 & 1078 & 52.7 & 1583 & 74.1 & 115 & 6.6 & 56 & 3.7 & 14 & 1.0 & 12,056 & 599.6 \\
\hline
\end{tabular}


Deprivation Index

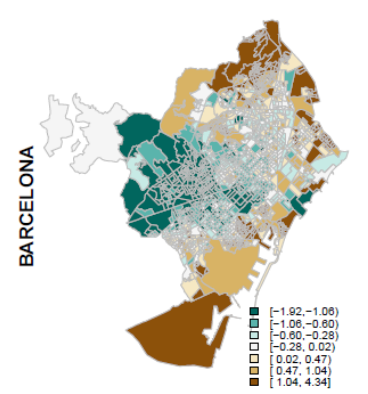

Deprivation Index

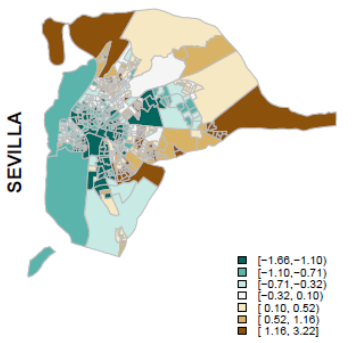

2001-2004

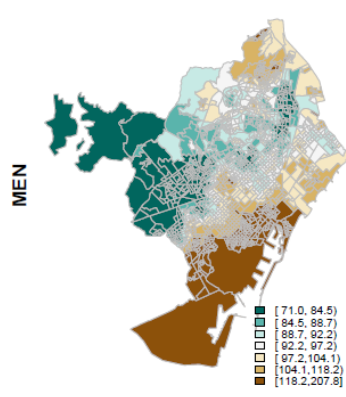

2001-2004

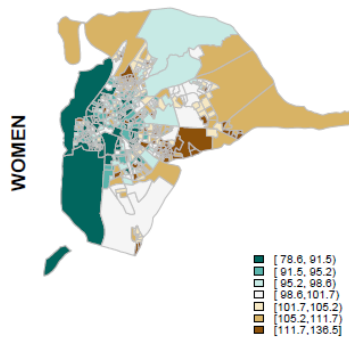

2005-2008

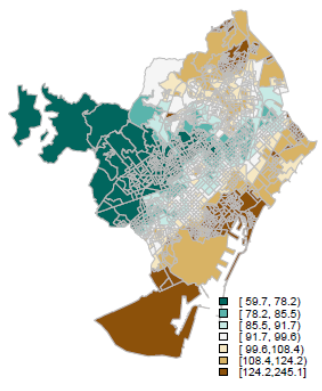

2005-2008

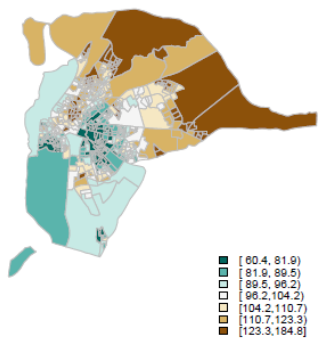

2009-2012

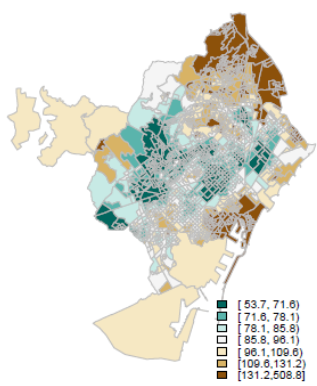

2009-2012

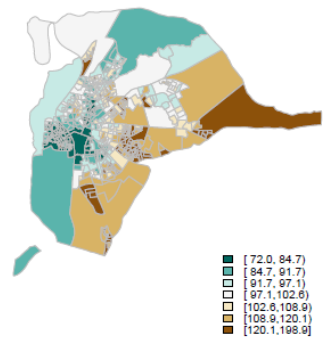

Figure 1. Deprivation index and Smootherd Standardized Mortality Ratios for the 3 periods among men in Barcelona and women in Sevilla.

Figure 2 and Table 3 show the association between the deprivation index and all-cause and cause-specific mortality in the three periods in men. In all cities, there was an association between the deprivation index and all-cause mortality that remained stable over the three periods. Regarding specific causes, in most cities there was a positive association between the deprivation index and mortality due to infectious and parasitic diseases that remained stable over the three periods. The same pattern was observed for mortality from cirrhosis, except in San Sebastian, where there was no association in the first $(\mathrm{RR}=1.55,95 \% \mathrm{CI}=0.52-3.39)$ and second $(\mathrm{RR}=1.69,95 \% \mathrm{CI}=0.75-3.31)$ periods but an association appeared in the third when residents of the most deprived census tracts were 6.49 times more likely to die of cirrhosis $(R R=6.49,95 \% C I=2.29-15.86)$ and this increase was significant. In relation to ischemic heart disease, an association was found between deprivation and mortality in Barcelona and Madrid that remained stable over time, and an association appeared in the third period when the risk of mortality from ischemic heart diseases was 1.85 ( $R R=1.85,95 \% \mathrm{CI}$ $=1.19-2.77)$ in Granada and 1.39 in $(R R=1.39,95 \% C I=1.06-1.80)$ Sevilla. In addition, there was an association between deprivation and mortality due to cerebrovascular disease in the second and third periods in Barcelona, while in Vitoria there was no association in the first $(\mathrm{RR}=1.39,95 \% \mathrm{CI}=0.84-2.16)$ and second ( $\mathrm{RR}=0.91,95 \% \mathrm{CI}=0.52-1.47)$ periods; however, in the third period, the residents of the most-deprived census tract were 1.91 times more likely to die of cerebrovascular diseases $(R R=1.91$, $95 \% \mathrm{CI}=1.18-2.93$ ) and this increase was significant. An association was found between deprivation and mortality due to suicide and that remained stable over the three periods only in Sevilla and there was an association in the third period in Madrid and San Sebastian. An association with diabetes appeared in the third period in Barcelona, Bilbao, Madrid, and Granada. Finally, most of the cities showed no association between deprivation and mortality due to traffic injuries in any of the periods. 

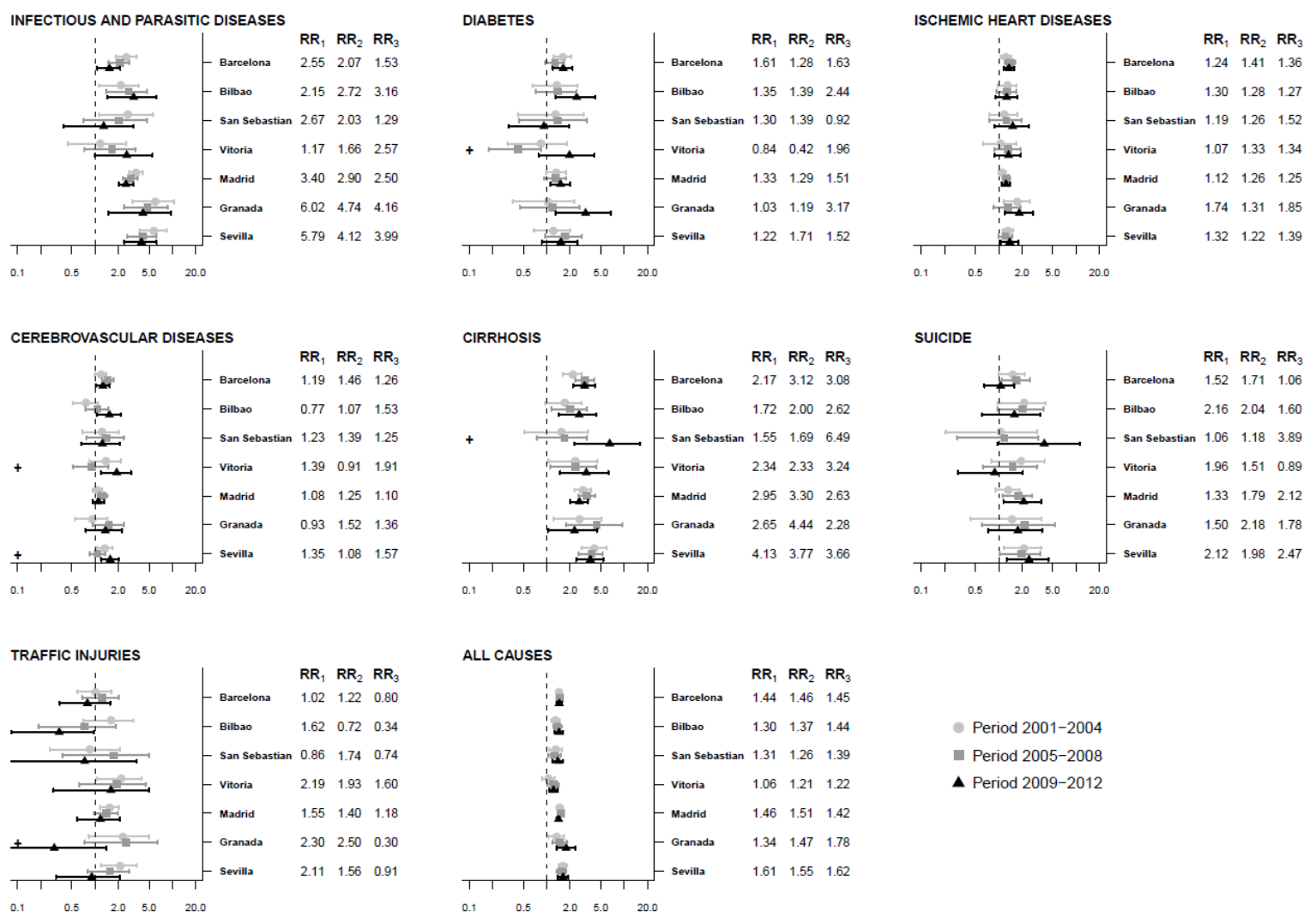

Figure 2. Association between the deprivation index and mortality. Relative risk comparing the 95th to 5 th percentiles of the deprivation index by period and cause of death in seven Spanish cities for men, 2001-2012. Note: + indicates that $R_{3}$ is statistically significantly different than $R R_{2}$. RR1: RR 2001-2004 period, RR2: RR 2005-2008 period, RR3: RR 2009-2012 period.

Figure 3 and Table 4 show the association between the deprivation index and cause-specific and all-cause mortality in the three periods in women. In Barcelona, Granada, and Sevilla, residents of most-deprived census tracts were more likely to die in the third period (which was significant for Barcelona and Granada). Regarding specific causes, in Barcelona, Madrid, and Sevilla, there was a positive association between the deprivation index and mortality due to infectious and parasitic diseases that remained stable over the three periods. The same pattern was observed for mortality due to diabetes, except there was no association in the first period in Sevilla, and in Granada there was no association in the second $(R R=1.75,95 \% C I=0.61-3.82)$ period but an association appeared in the third, when the residents of most-deprived census tracts were 4.20 times more likely to die of diabetes $(\mathrm{RR}=4.20,95 \% \mathrm{CI}=1.77-8.46)$. In relation to ischemic heart disease and cirrhosis, there was association between the deprivation index and mortality in Barcelona and Sevilla that remained stable over time. In addition, in Bilbao, there was no association between the index and mortality due to ischemic heart disease in the first $(\mathrm{RR}=1.17,95 \% \mathrm{CI}=0.81-1.64)$ and second $(\mathrm{RR}=1.27,95 \% \mathrm{CI}=$ $0.78-1.92)$ periods but an association appeared in the third period with a $\mathrm{RR}$ of 1.49 ( $\mathrm{RR}=1.49,95 \% \mathrm{CI}$ $=1.05-2.04)$. In addition, in most cities, there was no association between deprivation and mortality due to cerebrovascular diseases except in Granada, where there was association in the second and third periods. Finally, most of the cities showed no association between deprivation and mortality due to suicide and traffic injuries in any of the periods. 
Table 3. Association between mortality and the deprivation index. Relative risk comparing the 95th to 5th percentiles of the deprivation index by period and cause of death in 7 Spanish cities for men, 2001-2012.

\begin{tabular}{|c|c|c|c|c|c|c|c|c|c|c|c|c|c|c|c|c|c|}
\hline \multirow[b]{2}{*}{$\begin{array}{c}\text { Cities (No. Census } \\
\text { Tract) }\end{array}$} & \multirow[b]{2}{*}{ Period } & \multicolumn{2}{|c|}{$\begin{array}{c}\text { Infectious and } \\
\text { Parasitic Diseases }\end{array}$} & \multicolumn{2}{|c|}{ Diabetes } & \multicolumn{2}{|c|}{$\begin{array}{l}\text { Ischemic Heart } \\
\text { Diseases }\end{array}$} & \multicolumn{2}{|c|}{$\begin{array}{l}\text { Cerebrovascular } \\
\text { Diseases }\end{array}$} & \multicolumn{2}{|c|}{ Cirrhosis } & \multicolumn{2}{|c|}{ Suicide } & \multicolumn{2}{|c|}{ Traffic Injuries } & \multicolumn{2}{|c|}{ All Causes } \\
\hline & & $\mathbf{R R}$ & $95 \% \mathrm{CI}$ & $\mathbf{R R}$ & $95 \% \mathrm{CI}$ & RR & $95 \% \mathrm{CI}$ & RR & $95 \% \mathrm{CI}$ & $\mathbf{R R}$ & $95 \% \mathrm{CI}$ & $\mathbf{R R}$ & $95 \% \mathrm{CI}$ & $\mathbf{R R}$ & $95 \% \mathrm{CI}$ & $\mathbf{R R}$ & $95 \% \mathrm{CI}$ \\
\hline \multirow{3}{*}{ Barcelona (1491) } & 2001-2004 & 2.55 & $(1.85-3.41)$ & 1.61 & $(1.23-2.05)$ & 1.24 & $(1.08-1.44)$ & 1.19 & $(0.99-1.41)$ & 2.17 & $(1.60-2.86)$ & 1.52 & $(1.00-2.19)$ & 1.02 & $(0.59-1.63)$ & 1.44 & $(1.36-1.53)$ \\
\hline & 2005-2008 & 2.07 & $(1.51-2.79)$ & 1.28 & $(0.96-1.66)$ & 1.41 & $(1.22-1.64)$ & 1.46 & $(1.22-1.73)$ & 3.12 & $(2.31-4.11)$ & 1.71 & $(1.11-2.54)$ & 1.22 & $(0.68-2.02)$ & 1.46 & $(1.37-1.55)$ \\
\hline & 2009-2012 & 1.53 & $(1.07-2.10)$ & 1.63 & $(1.19-2.16)$ & 1.36 & $(1.16-1.59)$ & 1.26 & $(1.02-1.55)$ & 3.08 & $(2.18-4.16)$ & 1.06 & $(0.66-1.59)$ & 0.80 & $(0.35-1.56)$ & 1.45 & (1.35-1.55) \\
\hline \multirow[t]{3}{*}{ Bilbao (288) } & 2001-2004 & 2.15 & $(1.13-3.67)$ & 1.35 & $(0.65-2.46)$ & 1.30 & $(0.97-1.72)$ & 0.77 & $(0.53-1.10)$ & 1.72 & $(0.95-2.85)$ & 2.16 & $(0.98-4.05)$ & 1.62 & $(0.70-3.13)$ & 1.30 & (1.13-1.48) \\
\hline & $2005-2008$ & 2.72 & $(1.41-4.64)$ & 1.39 & $(0.72-2.45)$ & 1.28 & $(0.94-1.70)$ & 1.07 & $(0.76-1.49)$ & 2.00 & $(1.15-3.26)$ & 2.04 & $(0.96-3.79)$ & 0.72 & $(0.19-1.86)$ & 1.37 & (1.21-1.54) \\
\hline & 2009-2012 & 3.16 & $(1.46-6.12)$ & 2.44 & $(1.31-4.17)$ & 1.27 & $(0.90-1.74)$ & 1.53 & $(1.06-2.16)$ & 2.62 & $(1.43-4.35)$ & 1.60 & $(0.60-3.48)$ & 0.34 & $(0.08-0.96)$ & 1.44 & (1.27-1.63) \\
\hline \multirow[t]{3}{*}{ San Sebastian (140) } & 2001-2004 & 2.67 & $(1.11-5.61)$ & 1.30 & $(0.42-2.97)$ & 1.19 & $(0.76-1.75)$ & 1.23 & $(0.69-2.01)$ & 1.55 & $(0.52-3.39)$ & 1.06 & $(0.21-3.15)$ & 0.86 & $(0.26-2.09)$ & 1.31 & (1.07-1.58) \\
\hline & 2005-2008 & 2.03 & $(0.71-4.67)$ & 1.39 & $(0.44-3.30)$ & 1.26 & $(0.77-1.96)$ & 1.39 & $(0.77-2.34)$ & 1.69 & $(0.75-3.31)$ & 1.18 & $(0.29-3.27)$ & 1.74 & $(0.38-5.02)$ & 1.26 & (1.05-1.50) \\
\hline & 2009-2012 & 1.29 & $(0.40-3.09)$ & 0.92 & $(0.32-1.97)$ & 1.52 & $(0.89-2.44)$ & 1.25 & $(0.66-2.11)$ & 6.49 & $(2.29-15.86)$ & 3.89 & $(0.98-11.31)$ & 0.74 & $(0.04-3.45)$ & 1.39 & (1.19-1.62) \\
\hline \multirow{3}{*}{ Vitoria (168) } & 2001-2004 & 1.17 & $(0.44-2.51)$ & 0.84 & $(0.31-1.83)$ & 1.07 & $(0.63-1.68)$ & 1.39 & $(0.84-2.16)$ & 2.34 & $(1.05-4.54)$ & 1.96 & $(0.81-3.95)$ & 2.19 & (1.07-3.95) & 1.06 & $(0.87-1.26)$ \\
\hline & $2005-2008$ & 1.66 & $(0.72-3.30)$ & 0.42 & $(0.18-0.85)$ & 1.33 & $(0.87-1.94)$ & 0.91 & $(0.52-1.47)$ & 2.33 & $(1.11-4.39)$ & 1.51 & $(0.63-3.10)$ & 1.93 & $(0.62-4.50)$ & 1.21 & (1.02-1.43) \\
\hline & 2009-2012 & 2.57 & $(1.00-5.52)$ & 1.96 & $(0.78-4.14)$ & 1.34 & $(0.89-1.94)$ & 1.91 & $(1.18-2.93)$ & 3.24 & $(1.48-6.26)$ & 0.89 & $(0.30-2.07)$ & 1.60 & $(0.29-5.00)$ & 1.22 & $(1.07-1.39)$ \\
\hline \multirow{3}{*}{ Madrid (2358) } & 2001-2004 & 3.40 & $(2.76-4.16)$ & 1.33 & $(0.98-1.78)$ & 1.12 & $(1.00-1.26)$ & 1.08 & $(0.94-1.23)$ & 2.95 & $(2.30-3.72)$ & 1.33 & $(0.91-1.89)$ & 1.55 & $(1.16-2.03)$ & 1.46 & (1.39-1.53) \\
\hline & 2005-2008 & 2.90 & $(2.32-3.59)$ & 1.29 & $(0.91-1.77)$ & 1.26 & $(1.12-1.42)$ & 1.25 & $(1.08-1.43)$ & 3.30 & $(2.58-4.18)$ & 1.79 & $(1.15-2.67)$ & 1.40 & $(0.98-1.95)$ & 1.51 & (1.44-1.58) \\
\hline & 2009-2012 & 2.50 & $(2.01-3.09)$ & 1.51 & $(1.12-1.99)$ & 1.25 & $(1.10-1.41)$ & 1.10 & $(0.93-1.30)$ & 2.63 & $(2.02-3.38)$ & 2.12 & $-3.60)$ & 1.18 & $(0.59-2.09)$ & 1.42 & (1.35-1.49) \\
\hline \multirow[t]{3}{*}{ Granada (181) } & 2001-2004 & 6.02 & (3.08-10.47) & 1.03 & $(0.36-2.25)$ & 1.74 & $(1.15-2.51)$ & 0.93 & (0.54-1.44) & 2.65 & $(1.23-5.06)$ & 1.50 & $(0.43-3.61)$ & 2.30 & $(0.83-5.01)$ & 1.34 & (1.05-1.66) \\
\hline & $2005-2008$ & 4.74 & $(2.37-8.67)$ & 1.19 & $(0.45-2.61)$ & 1.31 & $(0.86-1.89)$ & 1.52 & $(0.93-2.37)$ & 4.44 & $(1.78-9.50)$ & 2.18 & $(0.61-5.36)$ & 2.50 & $(0.74-6.48)$ & 1.47 & (1.19-1.80) \\
\hline & 2009-2012 & 4.16 & $(1.48-9.56)$ & 3.17 & $(1.31-6.69)$ & 1.85 & $(1.19-2.77)$ & 1.36 & $(0.76-2.25)$ & 2.28 & $(1.03-4.43)$ & 1.78 & $(0.74-3.64)$ & 0.30 & $(0.01-1.41)$ & 1.78 & (1.33-2.33) \\
\hline \multirow[t]{3}{*}{ Sevilla (510) } & 2001-2004 & 5.79 & $(3.76-8.58)$ & 1.22 & $(0.67-2.00)$ & 1.32 & $(1.13-1.54)$ & 1.35 & $(1.05-1.70)$ & 4.13 & $(2.82-5.87)$ & 2.12 & $(1.18-3.55)$ & 2.11 & $(1.19-3.49)$ & 1.61 & (1.44-1.81) \\
\hline & 2005-2008 & 4.12 & $(2.62-6.19)$ & 1.71 & $(0.95-2.85)$ & 1.22 & $(0.98-1.51)$ & 1.08 & $(0.85-1.34)$ & 3.77 & $(2.60-5.32)$ & 1.98 & $(1.05-3.46)$ & 1.56 & $(0.81-2.74)$ & 1.55 & $(1.36-1.76)$ \\
\hline & 2009-2012 & 3.99 & $(2.39-6.29)$ & 1.52 & $(0.86-2.50)$ & 1.39 & $(1.06-1.80)$ & 1.57 & $(1.21-2.02)$ & 3.66 & $(2.38-5.38)$ & 2.47 & $(1.27-4.37)$ & 0.91 & $(0.31-2.09)$ & 1.62 & (1.38-1.89) \\
\hline
\end{tabular}


Table 4. Association between mortality and the deprivation index. Relative risk comparing the 95th to 5th percentiles of the deprivation index by period and cause of death in 7 Spanish cities for women, 2001-2012.

\begin{tabular}{|c|c|c|c|c|c|c|c|c|c|c|c|c|c|c|c|c|c|}
\hline \multirow[b]{2}{*}{$\begin{array}{c}\text { Cities (No. Census } \\
\text { Tract) }\end{array}$} & \multirow[b]{2}{*}{ Period } & \multicolumn{2}{|c|}{$\begin{array}{c}\text { Infectious and } \\
\text { Parasitic Diseases }\end{array}$} & \multicolumn{2}{|c|}{ Diabetes } & \multicolumn{2}{|c|}{$\begin{array}{l}\text { Ischemic Heart } \\
\text { Diseases }\end{array}$} & \multicolumn{2}{|c|}{$\begin{array}{c}\text { Cerebrovascular } \\
\text { Diseases }\end{array}$} & \multicolumn{2}{|c|}{ Cirrhosis } & \multicolumn{2}{|c|}{ Suicide } & \multicolumn{2}{|c|}{ Traffic Injuries } & \multicolumn{2}{|c|}{ All Causes } \\
\hline & & $\mathbf{R R}$ & $95 \%$ CI & $\mathbf{R R}$ & $95 \%$ CI & $\mathbf{R R}$ & $95 \% \mathrm{CI}$ & RR & $95 \% \mathrm{CI}$ & $\mathbf{R R}$ & $95 \%$ CI & $\mathbf{R R}$ & $95 \%$ CI & $\mathbf{R R}$ & $95 \%$ CI & $\mathbf{R R}$ & $95 \% \mathrm{CI}$ \\
\hline \multirow{3}{*}{ Barcelona (1491) } & 2001-2004 & 2.05 & $(1.54-2.66)$ & 2.13 & $(1.63-2.70)$ & 1.39 & $(1.17-1.62)$ & 1.13 & $(0.99-1.28)$ & 2.17 & $(1.54-2.97)$ & 0.89 & $(0.47-1.52)$ & 0.90 & $(0.46-1.56)$ & 1.13 & $(1.06-1.20)$ \\
\hline & 2005-2008 & 1.84 & $(1.40-2.40)$ & 1.71 & $(1.24-2.25)$ & 1.34 & $(1.14-1.56)$ & 1.01 & $(0.87-1.17)$ & 2.07 & $(1.43-2.89)$ & 0.77 & $(0.36-1.48)$ & 0.64 & $(0.23-1.45)$ & 1.08 & $(1.00-1.15)$ \\
\hline & 2009-2012 & 1.65 & $(1.23-2.17)$ & 1.95 & $(1.42-2.56)$ & 1.33 & $(1.10-1.61)$ & 1.05 & $(0.88-1.24)$ & 1.99 & $(1.36-2.81)$ & 1.16 & $(0.56-2.18)$ & 0.57 & $(0.18-1.37)$ & 1.19 & (1.10-1.29) \\
\hline \multirow[t]{3}{*}{ Bilbao (288) } & 2001-2004 & 1.96 & $(1.06-3.33)$ & 2.11 & $(1.20-3.39)$ & 1.17 & $(0.81-1.64)$ & 0.78 & $(0.54-1.09)$ & 1.82 & $(0.87-3.33)$ & 0.91 & $(0.17-2.69)$ & 2.69 & $(0.79-6.54)$ & 1.08 & $(0.91-1.25)$ \\
\hline & 2005-2008 & 1.90 & $(0.85-3.52)$ & 1.36 & $(0.72-2.30)$ & 1.27 & $(0.78-1.92)$ & 1.07 & $(0.81-1.40)$ & 2.35 & $(0.80-5.70)$ & 1.09 & $(0.37-2.56)$ & 0.78 & $(0.07-3.20)$ & 1.07 & $(0.91-1.24)$ \\
\hline & 2009-2012 & 1.11 & $(0.60-1.89)$ & 1.60 & $(0.90-2.65)$ & 1.49 & $(1.05-2.04)$ & 0.81 & $(0.59-1.09)$ & 1.43 & $(0.59-2.94)$ & 1.25 & $(0.29-3.52)$ & 2.00 & $(0.03-10.87)$ & 1.10 & $(0.97-1.24)$ \\
\hline \multirow[t]{3}{*}{ San Sebastian (140) } & 2001-2004 & 0.85 & $(0.27-1.97)$ & 1.74 & $(0.47-4.28)$ & 0.68 & $(0.33-1.21)$ & 0.95 & $(0.54-1.50)$ & 6.07 & $(1.50-16.39)$ & & & & & 0.76 & $(0.52-1.06)$ \\
\hline & 2005-2008 & 0.86 & $(0.28-1.98)$ & 2.05 & $(0.64-4.70)$ & 0.86 & $(0.41-1.52)$ & 0.72 & $(0.41-1.16)$ & 3.40 & (0.49-13.19) & & & & & 0.85 & $(0.60-1.14)$ \\
\hline & 2009-2012 & 1.89 & $(0.57-4.68)$ & 1.73 & $(0.59-3.78)$ & 0.65 & $(0.28-1.24)$ & 1.00 & $(0.58-1.59)$ & 2.72 & $(0.72-7.19)$ & & & & & 0.96 & $(0.75-1.21)$ \\
\hline \multirow{3}{*}{ Vitoria (168) } & 2001-2004 & 1.79 & $(0.72-3.68)$ & 0.99 & $(0.44-1.91)$ & 1.12 & $(0.61-1.89)$ & 0.75 & $(0.44-1.15)$ & 3.90 & (1.22-9.68) & 1.21 & $(0.17-4.08)$ & 1.01 & $(0.18-3.07)$ & 0.78 & $(0.61-0.98)$ \\
\hline & $2005-2008$ & 0.80 & $(0.33-1.64)$ & 0.78 & $(0.37-1.44)$ & 1.13 & $(0.57-2.00)$ & 0.81 & $(0.50-1.25)$ & 4.23 & $(1.06-11.86)$ & 0.39 & $(0.02-1.71)$ & 3.59 & $(0.35-14.17)$ & 0.80 & $(0.63-1.00)$ \\
\hline & 2009-2012 & 0.72 & $(0.22-1.67)$ & 0.63 & $(0.27-1.22)$ & 1.74 & $(1.07-2.67)$ & 1.20 & $(0.79-1.75)$ & 1.65 & $(0.48-4.19)$ & 0.32 & $(0.02-1.39)$ & 2.06 & $(0.08-10.08)$ & 0.91 & $(0.77-1.07)$ \\
\hline \multirow{3}{*}{ Madrid (2358) } & 2001-2004 & 1.66 & $(1.28-2.11)$ & 1.55 & $(1.22-1.93)$ & 0.95 & $(0.83-1.07)$ & 0.92 & $(0.81-1.05)$ & 1.57 & $(1.17-2.07)$ & 0.89 & $(0.49-1.50)$ & 0.84 & $(0.51-1.27)$ & 1.02 & $(0.97-1.08)$ \\
\hline & 2005-2008 & 1.75 & $(1.39-2.17)$ & 1.38 & $(1.04-1.80)$ & 1.10 & $(0.96-1.25)$ & 0.93 & $(0.81-1.07)$ & 1.34 & $(0.92-1.88)$ & 0.70 & $(0.32-1.35)$ & 1.76 & $(0.95-2.97)$ & 1.08 & $(1.03-1.14)$ \\
\hline & 2009-2012 & 1.57 & (1.26-1.95) & 1.67 & $(1.29-2.11)$ & 1.08 & $(0.93-1.25)$ & 0.92 & $(0.80-1.06)$ & 0.95 & $(0.62-1.38)$ & 2.12 & $(0.56-5.74)$ & 2.19 & $(0.69-5.20)$ & 1.04 & $(0.98-1.10)$ \\
\hline \multirow[t]{3}{*}{ Granada (181) } & 2001-2004 & 3.32 & $(1.26-7.01)$ & 4.13 & $(1.97-7.54)$ & 1.38 & $(0.88-1.99)$ & 1.34 & $(0.84-1.96)$ & 3.11 & (1.19-6.52) & 0.40 & $(0.04-1.48)$ & & & 0.87 & $(0.60-1.20)$ \\
\hline & $2005-2008$ & 2.13 & $(0.83-4.45)$ & 1.75 & $(0.61-3.82)$ & 1.97 & $(1.30-2.88)$ & 1.72 & $(1.12-2.50)$ & 1.54 & $(0.47-3.85)$ & 1.05 & $(0.21-3.17)$ & & & 1.00 & $(0.76-1.26)$ \\
\hline & 2009-2012 & 2.14 & $(0.69-5.09)$ & 4.20 & $(1.77-8.46)$ & 1.49 & $(0.93-2.28)$ & 1.64 & $(1.00-2.55)$ & 2.61 & $(0.81-6.56)$ & 1.08 & $(0.13-4.06)$ & & & 1.72 & $(1.31-2.22)$ \\
\hline \multirow[t]{3}{*}{ Sevilla (510) } & 2001-2004 & 1.88 & $(1.10-3.00)$ & 1.50 & $(0.92-2.27)$ & 1.66 & $(1.36-1.99)$ & 0.93 & $(0.72-1.17)$ & 2.49 & $(1.24-4.47)$ & 0.33 & $(0.08-0.87)$ & 1.37 & $(0.41-3.38)$ & 1.08 & (0.95-1.22) \\
\hline & $2005-2008$ & 2.49 & $(1.44-4.03)$ & 1.75 & $(1.07-2.69)$ & 1.45 & $(1.09-1.91)$ & 0.97 & $(0.77-1.18)$ & 2.14 & $(1.05-3.93)$ & 1.41 & $(0.45-3.32)$ & 1.92 & $(0.32-6.38)$ & 1.11 & $(0.97-1.27)$ \\
\hline & 2009-2012 & 1.72 & $(1.03-2.68)$ & 1.70 & $(1.04-2.64)$ & 1.60 & $(1.23-2.03)$ & 1.23 & $(0.96-1.54)$ & 3.50 & $(1.73-6.27)$ & 1.39 & $(0.45-3.29)$ & 0.73 & $(0.05-3.32)$ & 1.17 & (1.01-1.35) \\
\hline
\end{tabular}



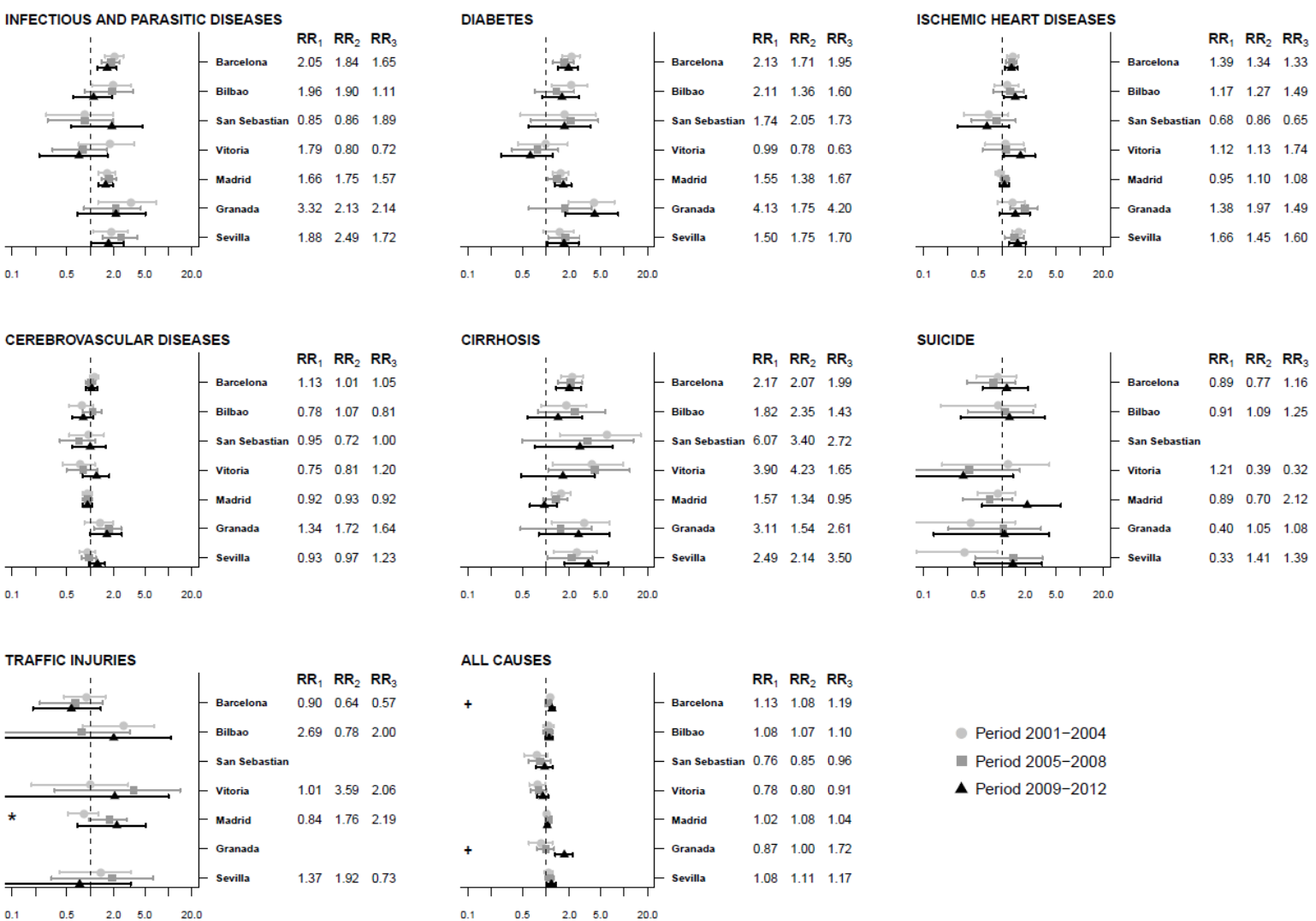

Figure 3. Association between the deprivation index and mortality. Relative risk comparing the 95th to 5th percentiles of the deprivation index by period and cause of death in seven Spanish cities for women, 2001-2012. Note: * indicates that $R_{2}$ is statistically significantly different than $R_{1}$ and + indicates that $R_{3}$ is statistically significantly different than $R_{2}$. RR1: RR 2001-2004 period, RR2: RR 2005-2008 period, RR3: RR 2009-2012 period.

\section{Discussion}

\subsection{Main Findings of the Study}

In all cities, in both men and women, mortality rates for most of the causes analyzed tended to decrease in the three periods studied, including the period of the financial crisis. In general, socioeconomic inequalities were found for many of the causes analyzed, especially in men. Among women, inequalities were less important. In some cities, and for some specific causes of death, previously absent inequalities appeared in the crisis period, especially among men. This suggests a possible increase in mortality inequalities for some specific causes associated with the crisis, and perhaps, additionally associated with specific local contexts.

\subsection{What Is Already Known and What Does This Study Add?}

Studies conducted in Europe have found that the crisis was associated with a decrease in all-cause mortality and mortality from certain specific causes, such as cardiovascular disease, cirrhosis, infectious diseases, and traffic injuries [12-14,18,20], and with an increase in mortality due to suicide [12-16,20, 35-38]. Our results are in line with those of these studies, except for mortality due to suicide, which in this study continued to decrease after the start of the financial crisis. Studies performed in Spain in the first years of the crisis [18] have reported the same decreasing tendency. However, more recent reports have shown that mortality due to suicide increased after the start of the crisis, especially in men $[39,40]$. Moreover, two studies based on urban areas did not show an increase in inequalities in suicide mortality during the crisis period [41,42]. 
Of note, the results found can be partially explained by the different incubation periods of the diseases. Suicide mortality can be an effect of the job loss and financial strain due to the economic crisis [11,12]. Infectious diseases also have a short incubation period and their incidence has risen in some countries during the crisis [7], but in our study this is not reflected in mortality data. Other relevant factors are the mechanisms that explain the changes in health outcomes during the crisis period for each cause of mortality. Catalano et al. (2011) [11] explains how the stress mechanism due to unemployment and job loss can increase unhealthy behaviors as a coping measure, such as alcohol intake or other drug use, which can be related to some diseases.

Our study demonstrates the existence of socioeconomic inequalities in all-cause mortality that remained stable throughout the study period in men and increased in two cities among women. A study conducted in Finland in the 1990s reported that mortality followed the same tendency after the start of the financial crisis [41]. However, several studies have reported that inequalities increased in periods of economic crisis, as we found for women in Barcelona and Granada $[4,24,43]$.

Previous studies have detected socioeconomic inequalities in mortality due to ischemic heart disease and cerebrovascular disease in periods prior to the onset of the crisis in small areas in various European cities [25]. Some studies have found socioeconomic inequalities in mortality due to cardiovascular disease (ischemic heart disease and cerebrovascular disease) that persisted after the onset of the financial crisis [44,45]. This result is in agreement with our findings for most of the cities in our study.

In periods before the crisis, socioeconomic inequalities in mortality have been found in mortality due to traffic injuries in small areas of several Spanish [28] and European [46] cities, especially in men. In agreement with our results, subsequent studies have found no socioeconomic inequalities due to this cause after the start of the crisis [45].

We have found no studies analyzing the effects of the financial crisis on socioeconomic inequalities in mortality due to infectious diseases, diabetes, or cirrhosis. However, some studies have reported the existence of these inequalities in periods before the onset of the crisis [25,27].

Most causes of death on social inequalities in mortality seem to differ in men and women, being less important in women. This could be explained by the distinct distribution of risk factors between men and women as a result of socially accepted differences in gender-related roles and powers. Traditional gender roles produce greater pressure on men in terms of earnings and unemployment and mainly on those from less advantageous social classes. Moreover, hegemonic masculinity is more closely related to risky health behaviors [47,48]. Total mortality increased in Barcelona and Granada among women during the economic crisis, although death rates and the increase in the risk of death in the third period were much higher in Granada, a city located in the south of Spain where the crisis has been more keenly experienced [49].

As shown, it is not easy to quantify the effects of the financial crisis on mortality and it is even more difficult to quantify those of socioeconomic inequalities in mortality. Moreover, the results of this study and those in the literature are inconclusive and sometimes contradictory. Some of the factors that could explain the discrepant results are the following: the heterogeneity of the socioeconomic variables analyzed, the type of measures used to analyze the magnitude of social inequalities in health, and the various state social policies implemented during the crisis [4]. In Spain, the contribution of social policies and austerity on health results is still controversial [4,21,45,50-52]. It is worth mentioning that Spain has a national health system that can protect the treatment of population diseases. This system suffered from austerity measures, but in our study, it is difficult to discern the possible influence of health budget cuts on the increase in inequalities, given that our data were gathered until 2012 and these policies only began to be implemented in 2011. However, the effect of the economic crisis in Spain has been heterogeneous in the different autonomous communities, which may be because each region has some control over how socials cuts are implemented [4,7]. Another important factor is the diversity of time periods studied. The use of short periods for the analyses makes it difficult to capture the long-term effects on mortality inequalities. It is reasonable to expect that the effects of 
socioeconomic circumstances on mortality will not be contemporaneous but will become apparent in short and long-term effects. Finally, another factor is the changes in the socioeconomic groups compared during the periods. In Spain, since 2000, the foreign-born population has increased, although this migratory flow decreased after the beginning of the financial crisis [53]. The growth of immigration could present residential segregation patterns because immigrants from low-income countries tend to increase in socioeconomically disadvantaged neighborhoods [54]. In this regard, some studies have found that the increase in the foreign-born population could explain the decrease in mortality inequalities between more and less-disadvantaged areas [53,55].

\subsection{Limitations of This Study}

The main limitation of the present study is the use of a single deprivation index in the three periods studied. Although the socio-economic situation may have changed over the period studied, we believe that the distribution of deprivation at the small area level has not undergone substantial variations over time because the processes involving changes in socioeconomic deprivation at area level are slow [56]. To provide information on the influence of the financial crisis on socioeconomic inequalities in health, this study analyzed the possible effect of the crisis on socioeconomic inequalities in various causes of mortality in seven Spanish cities with different economic contexts. Moreover, until 2012, mortality statistics in Spain did not incorporate information on the educational attainment of the deceased, and consequently ecological studies were needed to analyze socioeconomic inequalities in mortality. In this context, it is important to stress that small area studies are those that most closely approximate individual-level studies and are least susceptible to the component of ecological bias created by heterogeneity within areas of exposure or other determining factors [57]. Moreover, they allow detection of geographical patterns in mortality and deprivation that might not be evident in larger geographical areas. Finally, in some cities, it was not possible to analyze all specific causes due to the small numbers of deaths observed. The low number of deaths may have led to the low statistical power of the data to detect significant associations. However, the statistical method chosen is intended to reduce the impact this may have on the results.

\section{Conclusions}

This study shows that socioeconomic inequalities in mortality in small urban areas remained stable after the onset of the financial crisis for all-cause mortality, especially in men. Among women, inequalities were less important. In some cities, and for some specific causes of death, inequalities appeared in the crisis period, which had been absent in the previous periods, especially among men. In addition, there is evidence that economic crises have an impact on some health determinants such as the deterioration of the labor market, the difficulty of accessing housing, worse health behaviors, etc. In future, it will be necessary to continue to monitor the population's health and its determinants, and invest in information systems that enhance knowledge of the effect of economic crises on the health and wellbeing of the population. Moreover, further studies are needed on the effects of economic crises on health and inequalities in health, since multiple indicators can reflect negative effects in the long-term. Finally, it is important to stress that the policy response in managing the financial crises can mitigate or magnify the negative impacts on health and health inequalities. Therefore, financial crises provide a good opportunity to mainstream health in all policies and promote intersectional policies to improve the health of the population.

Author Contributions: All authors made substantial contributions to conception and design of this study. M.G., L.P. and M.M.-D. performed data analysis. All authors contributed to the interpretation of data. M.G., J.F. and C.B. were involved in drafting the manuscript and the rest of the authors revised it critically for important intellectual content. All authors have read and agreed to the published version of the manuscript.

Funding: This research was partially funded by the project entitled, "Efectos de la crisis en la salud de la población y sus determinantes en España" (PI13/00897), funded by the Instituto de Salud Carlos III (co-funded by the European Regional Development Fund). 
Conflicts of Interest: The authors declare no conflict of interest.

Ethics Approval and Consent to Participate: This article only uses secondary data.

\section{References}

1. Cortés-Franch, I.; González López-Valcárcel, B. Crisis económico-financiera y salud en España. Evidencia y perspectivas. Informe SESPAS 2014. Gac. Sanit. 2014, 28, 1-6. [CrossRef] [PubMed]

2. Quaglio, G.; Karapiperis, T.; Van Woensel, L.; Arnold, E.; McDaid, D. Austerity and health in Europe. Health Policy 2013, 113, 13-19. [CrossRef] [PubMed]

3. Dávila Quintana, C.D.; González López-Valcárcel, B. [The economic crisis and health]. Gac. Sanit. 2009, 23, 261-265. [CrossRef] [PubMed]

4. Bacigalupe, A.; Escolar-Pujolar, A. The impact of economic crises on social inequalities in health: What do we know so far? Int. J. Equity Health 2014, 13, 52. [CrossRef] [PubMed]

5. Bezruchka, S. The effect of economic recession on population health. Cmaj 2009, 181, 281-285. [CrossRef]

6. Budhdeo, S.; Watkins, J.; Atun, R.; Williams, C.; Zeltner, T.; Maruthappu, M. Changes in government spending on healthcare and population mortality in the European union, 1995-2010: A cross-sectional ecological study. J. R. Soc. Med. 2015, 108, 490-498. [CrossRef]

7. Bacigalupe, A.; Shahidi, F.V.; Muntaner, C.; Martín, U.; Borrell, C. Why is There so Much Controversy Regarding the Population Health Impact of the Great Recession? Reflections on Three Case Studies. Int. J. Health Serv. 2015, 46, 5-35. [CrossRef]

8. Falagas, M.E.; Vouloumanou, E.K.; Mavros, M.N.; Karageorgopoulos, D.E. Economic crises and mortality: A review of the literature. Int. J. Clin. Pract. 2009, 63, 1128-1135. [CrossRef]

9. Segura Benedicto, A. Recortes, austeridad y salud. Informe SESPAS 2014. Gac. Sanit. 2014, $28,7-11$. [CrossRef]

10. Suhrcke, M.; Stuckler, D. Will the recession be bad for our health? It depends. Soc. Sci. Med. 2012, 74, 647-653. [CrossRef]

11. Catalano, R.; Goldman-Mellor, S.; Saxton, K.; Margerison-Zilko, C.; Subbaraman, M.; LeWinn, K.; Anderson, E. The health effects of economic decline. Annu. Rev. Public Health 2011, 32, 431-450. [CrossRef] [PubMed]

12. Parmar, D.; Stavropoulou, C.; Ioannidis, J.P.A. Health outcomes during the 2008 financial crisis in Europe: Systematic literature review. Br. Med. J. 2008, 354, 1-11. [CrossRef] [PubMed]

13. Toffolutti, V.; Suhrcke, M. Assessing the short term health impact of the Great Recession in the European Union: A cross-country panel analysis. Prev. Med. (Baltim) 2014, 64, 54-62. [CrossRef] [PubMed]

14. Tapia Granados, J.A.; Rodriguez, J.M. Health, economic crisis, and austerity: A comparison of Greece, Finland and Iceland. Health Policy (New York) 2015, 119, 941-953. [CrossRef] [PubMed]

15. Coope, C.; Gunnell, D.; Hollingworth, W.; Hawton, K.; Kapur, N.; Fearn, V.; Metcalfe, C. Suicide and the 2008 economic recession: Who is most at risk? Trends in suicide rates in England and Wales 2001-2011. Soc. Sci. Med. 2014, 117, 76-85. [CrossRef] [PubMed]

16. Haw, C.; Hawton, K.; Gunnell, D.; Platt, S. Economic recession and suicidal behaviour: Possible mechanisms and ameliorating factors. Int. J. Soc. Psychiatry 2015, 61, 73-81. [CrossRef]

17. Stuckler, D.; Basu, S.; Suhrcke, M.; Coutts, A.; McKee, M. Effects of the 2008 recession on health: A first look at European data. Lancet 2011, 378, 124-125. [CrossRef]

18. Regidor, E.; Barrio, G.; Bravo, M.J.; de la Fuente, L. Has health in Spain been declining since the economic crisis? J. Epidemiol. Community Health 2014, 68, 280-282. [CrossRef]

19. Borrell, C.; Rodríguez-Sanz, M.; Malmusi, D.; Bartoll, X.; Novoa, A.M. The suffering of the population in the economic crisis of the Spanish State. Salud Colect. 2014, 10, 95-98. [CrossRef]

20. Karanikolos, M.; Heino, P.; McKee, M.; Stuckler, D.; Legido-Quigley, H. Effects of the global financial crisis on health in high-income OECD countries: A narrative review. Int. J. Health Serv. 2016, 46, 208-240. [CrossRef]

21. Lopez-Valcarcel, B.G.; Barber, P. Economic Crisis, Austerity Policies, Health and Fairness: Lessons Learned in Spain. Appl. Health Econ. Health Policy 2017, 15, 13-21. [CrossRef] [PubMed]

22. Bartoll, X.; Palencia, L.; Malmusi, D.; Suhrcke, M.; Borrell, C. The evolution of mental health in Spain during the economic crisis. Eur. J. Public Health 2014, 24, 415-418. [CrossRef] [PubMed] 
23. Rodríguez-Fonseca, M.; Palència, L.; Marí-Dell'Olmo, M.; Gandarillas, A.; Domínguez-Berjón, M.F.; Gotsens, M.; Borrell, C. Evolution of socio-economic inequalities in mortality in small geographical areas of the two largest cities in Spain (Barcelona and Madrid), 1996-2007. Public Health 2013, 127, 916-921. [CrossRef] [PubMed]

24. Maynou, L.; Saez, M.; Lopez-Casasnovas, G. Has the economic crisis widened the intraurban socioeconomic inequalities in mortality? The case of Barcelona, Spain. J. Epidemiol. Community Health 2016, 70, 114-124. [CrossRef] [PubMed]

25. Marí-Dell'Olmo, M.; Gotsens, M.; Palència, L.; Burström, B.; Corman, D.; Costa, G.; Gandarillas, A. Socioeconomic inequalities in cause-specific mortality in 15 European cities. J. Epidemiol. Community Health 2015, 69, 432-441. [CrossRef]

26. Santana, P.; Costa, C.; Marí-Dell'Olmo, M.; Gotsens, M.; Borrell, C. Mortality, material deprivation and urbanization: Exploring the social patterns of a metropolitan area. Int. J. Equity Health 2015, 14, 55. [CrossRef]

27. Borrell, C.; Marí-Dell'Olmo, M.; Serral, G.; Martínez-Beneito, M.; Gotsens, M. Inequalities in mortality in small areas of eleven Spanish cities (the multicenter MEDEA project). Health Place 2010, 16, 703-711. [CrossRef]

28. Gotsens, M.; Marí-Dell’Olmo, M.; Martínez-Beneito, M.A.; Pérez, K.; Pasarín, M.I.; Daponte, A.; Gandarillas, A. Socio-economic inequalities in mortality due to injuries in small areas of ten cities in Spain (MEDEA Project). Accid Anal Prev. 2011, 43, 1802-1810. [CrossRef]

29. Pérez, G.; Gotsens, M.; Palència, L.; Marí-Dell’Olmo, M.; Domínguez-Berjón, M.F.; Rodríguez-Sanz, M.; Bacigalupe, A. Protocolo del estudio sobre el efecto de la crisis económica en la mortalidad, la salud reproductiva y las desigualdades en salud en España. Gac. Sanit. 2016, 30, 472-476. [CrossRef]

30. INE. Censos de Población y Viviendas. Available online: http://www.ine.es/censo2001/internet.htm (accessed on 3 February 2020).

31. Domínguez-Berjón, M.F.; Borrell, C.; Cano-Serral, G.; Esnaola, S.; Nolasco, A.; Pasarín, M.I.; Escolar-Pujolar, A. Construcción de un índice de privación a partir de datos censales en grandes ciudades españolas (Proyecto MEDEA). Gac. Sanit. 2008, 22, 179-187. [CrossRef]

32. Besag, J.; York, J.; Mollié, A. Bayesian image restoration, with two applications in spatial statistics. Ann. Inst. Stat. Math. 1991, 43, 1-59. [CrossRef]

33. R. Team. R: A Language and Environment for Statistical Computing; R Foundation for Statistical Computing: Vienna, Austria, 2012.

34. Rue, H.; Martino, S. INLA: Functions Which Allow to Perform a Full Bayesian Analysis of Structured Additive Models Using Integrated Nested Laplace Approximation. R Package Version 0.0. 2009. Available online: https://www.r-inla.org (accessed on 23 January 2020).

35. Barr, B.; Taylor-Robinson, D.; Scott-Samuel, A.; McKee, M.; Stuckler, D. Suicides associated with the 2008-10 economic recession in England: Time trend analysis. BMJ 2012, 345, e5142. [CrossRef] [PubMed]

36. Baumbach, A.; Gulis, G. Impact of financial crisis on selected health outcomes in Europe. Eur. J. Public Health 2014, 24, 399-403. [CrossRef] [PubMed]

37. Laanani, M.; Ghosn, W.; Jougla, E.; Rey, G. Impact of unemployment variations on suicide mortality in Western European countries (2000-2010). J. Epidemiol. Community Health 2015, 69, 103-109. [CrossRef] [PubMed]

38. Santana, P.; Costa, C.; Cardoso, G.; Loureiro, A.; Ferrão, J. Suicide in Portugal: Spatial determinants in a context of economic crisis. Heal Place. Health Place 2015, 35, 85-94. [CrossRef]

39. Isabel, R.P.; Miguel, R.B.; Antonio, R.G.; Oscar, M.G. Economic crisis and suicides in Spain. Socio-demographic and regional variability. Eur. J. Health Econ. 2016, 18, 313-320. [CrossRef]

40. Alvarez-Galvez, J.; Salinas-Perez, J.A.; Rodero-Cosano, M.L.; Salvador-Carulla, L. Methodological barriers to studying the association between the economic crisis and suicide in Spain. BMC Public Health 2017, 17, 694. [CrossRef]

41. Borrell, C.; Marí-Dell'Olmo, M.; Gotsens, M.; Calvo, M.; Rodríguez-Sanz, M.; Bartoll, X.; Esnaola, S. Socioeconomic inequalities in suicide mortality before and after the economic recession in Spain. BMC Public Health 2017, 17, 772. [CrossRef]

42. Borrell, C.; Palència, L.; Marí Dell'Olmo, M.; Morrisson, J.; Deboosere, P.; Gotsens, M.; Rodríguez-Sanz, M. Socioeconomic inequalities in suicide mortality in European urban areas before and during the economic recession. Eur. J. Public Health 2019, 30, 92-98. [CrossRef] 
43. Ruiz-Ramos, M.; Córdoba-Doña, J.A.; Bacigalupe, A.; Juárez, S.; Escolar-Pujolar, A. Crisis económica al inicio del siglo XXI y mortalidad en España. Tendencia e impacto sobre las desigualdades sociales. Informe SESPAS 2014. Gac. Sanit. 2014, 28 (Suppl. S1), 89-96. [CrossRef]

44. Valkonen, T. Changes in socioeconomic inequalities in mortality during an economic boom and recession among middle-aged men and women in Finland. Eur. J. Public Health 2000, 10, 274-280. [CrossRef]

45. Regidor, E.; Vallejo, F.; Granados, J.A.T.; Viciana-Fernández, F.J.; de la Fuente, L.; Barrio, G. Mortality decrease according to socioeconomic groups during the economic crisis in Spain: A cohort study of 36 million people. Lancet 2016, 388, 2642-2652. Available online: http://inkinghub.elsevier.com/retrieve/pii/S0140673616304469 (accessed on 3 February 2020). [CrossRef]

46. Gotsens, M.; Marí-Dell'Olmo, M.; Pérez, K.; Palència, L.; Martinez-Beneito, M.-A.; Rodríguez-Sanz, M.; Dzúrová, D. Socioeconomic inequalities in injury mortality in small areas of 15 European cities. Health Place 2013, 24, 165-172. [CrossRef]

47. Courtenay, W.H. Constructions of masculinity and their influence on men's well-being: A theory of gender and health. Soc. Sci. Med. 2000, 50, 1385-1401. [CrossRef]

48. Möller-Leimkühler, A.M. The gender gap in suicide and premature death or: Why are men so vulnerable? Eur. Arch. Psychiatry Clin Neurosci. 2003, 253, 1-8. [CrossRef] [PubMed]

49. CCOO de Industria. Los efectos de la crisis en el estado de las autonomías; CCOO de Industria: Madrid, Spain, 2016.

50. Regidor, E.; Vallejo, F.; Granados, J.A.T.; Viciana-Fernández, F.J.; de la Fuente, L.; Barrio, G. Mortality decrease according to socioeconomic groups-Authors' reply. Lancet 2017, 389, 1795. [CrossRef]

51. Moya, A.Z.; Buffel, V.; Navarro Yáñez, C.J.; Bracke, P. Social inequality in morbidity, framed within the current economic crisis in Spain. Int. J. Equity Health 2015, 14, 131. [CrossRef]

52. Maynou, L.; Saez, M. Economic crisis and health inequalities: Evidence from the European Union. Int. J. Equity Health 2016, 15, 135. [CrossRef]

53. Rodríguez-Sanz, M.; Gotsens, M.; Marí-Dell'Olmo, M.; Mehdipanah, R.; Borrell, C. Twenty years of socioeconomic inequalities in premature mortality in Barcelona: The influence of population and neighbourhood changes. Health Place 2016, 39, 142-152. [CrossRef]

54. Nello, O. The challenges of urban renewal. Ten lessons from the Catalan experience. Anal. Soc. 2010.

55. Norman, P.; Boyle, P.; Exeter, D.; Feng, Z.; Popham, F. Rising premature mortality in the UK's persistently deprived areas: Only a Scottish phenomenon? Soc. Sci. Med. 2011, 73, 1575-1584. [CrossRef] [PubMed]

56. Thomson, H. A dose of realism for healthy urban policy: Lessons from area-based initiatives in the UK. J. Epidemiol. Community Health 2008, 62, 932-936. [CrossRef] [PubMed]

57. Richardson, S.; Thomson, A.; Best, N.; Elliott, P. Interpreting posterior relative risk estimates in disease-mapping studies. Environ. Health Perspect. 2004, 112, 1016-1025. [CrossRef] [PubMed] 